\title{
Embryonic and Postnatal Injections of Bromodeoxyuridine Produce Age-Dependent Morphological and Behavioral Abnormalities
}

\author{
Bryan Kolb, Brian Pedersen, Mark Ballermann, Robbin Gibb, and lan Q. Whishaw \\ Department of Psychology and Neuroscience, University of Lethbridge, Lethbridge, Canada T1K 3M4
}

The mitotic marker 5-bromodeoxyuridine (BrdU) was injected twice daily $(60 \mathrm{mg} / \mathrm{kg})$ into pregnant hooded rats on one of embryonic days $(E) 11,12,13,15,17$, or 21 , or into rat pups on postnatal day $(P)$ 10. The principal findings were the following: (1) BrdU exposure on E11 produces profound effects on body morphology, and animals must be fed a special diet because of chronic tooth abnormalities; (2) BrdU exposure at E17 or earlier produces a change in coat spotting pattern, the precise pattern varying with age; (3) BrdU exposure on E15 or earlier produces a reduction in both brain and body weight; (4) BrdU exposure on E17 or earlier reduces cortical thickness; (5) BrdU exposure on $\mathrm{E} 11-\mathrm{E} 13$ and at $\mathrm{P} 10$ reduces cerebellar size relative to cerebral size; (6) spatial learning is significantly affected after injections of BrdU at E11-E17, but the largest effect is on E17; (7) the deficit in spatial learning may be related in part to a reduction in visual acuity; and (8) skilled forelimb ability is most disrupted after BrdU exposure at E15 but is also impaired after injections on E13 or earlier. BrdU thus has teratological effects on body, brain, and behavior that vary with the developmental age of the fetus or infant.

Key words: bromodeoxyuridine; cerebral cortex; spatial learning; visual acuity; cerebellum; teratology
5-Bromodeoxyuridine (BrdU) is a thymidine analog that is incorporated into the DNA as 5-bromouracil during the $\mathrm{S}$ phase of the cell cycle of any cell. It is widely used as a mitotic marker in developmental work. The structure of BrdU is comparable to thymidine except that a bromine replaces the methyl group at carbon 5 of the thymine portion (Iball et al., 1966). Antibodies to BrdU allow for immunohistochemical labeling of cells that are replicating DNA at the time BrdU is available, and this has been shown to be an adequate labeling method (Dolbeare, 1994). BrdU competes with thymidine for sites on the replicating DNA strand, and in culture there can be almost total substitution of BrdU for thymidine in the nuclear DNA (Bick and Davidson, 1974). At various concentrations, BrdU has been found to produce various cellular abnormalities both in vitro (Stockdale et al., 1964; Wilt and Anderson, 1972; Trencer and Brachet, 1973; Agnish and Kochhar, 1976a,b; Pollard et al., 1976; Barasch and Bressler, 1977; Dribin and Jacobson, 1978) and in vivo (DiPaolo, 1964; Meller et al., 1973; Webster et al., 1973; Younkin and Silberberg, 1973; Yu, 1976; Bannigan and Langman, 1979; Shah et al., 1991; Nagao et al., 1997).

In the course of studying the effect of early cortical lesions on the production of astrocytes and neurons in the cortex of rats, we exposed developing embryos to BrdU on embryonic day (E) 13 (Kolb et al., 1998). The animals were allowed to grow until adulthood for behavioral investigations. The animals had clear behavioral abnormalities, both in studies of spatial learning and motor behavior, and postmortem analysis showed the brain weight of BrdU-treated rats to be $\sim 10 \%$ lower than that of untreated rats. Because BrdU is so widely used as a mitotic

\footnotetext{
Received Oct. 22, 1998; revised Dec. 23, 1998; accepted Jan. 5, 1999.

This research was supported by Medical Research Council of Canada grants to B.K. and I.Q.W. B.P. and M.B. were supported by an Alberta Heritage Foundation for Medical Research studentships. We thank Reed Kindt for photographic help.

Correspondence should be addressed to Dr. Bryan Kolb, Department of Psychology and Neuroscience, University of Lethbridge, Lethbridge, AB, Canada, T1K 3M4.

Copyright (C) 1999 Society for Neuroscience $\quad 0270-6474 / 99 / 192337-10 \$ 05.00 / 0$
}

marker in developmental work, we decided to systematically investigate the effect of embryonic BrdU treatment on behavior and cerebral morphology. Despite the extensive literature on the effects of BrdU on brain and body development, there have been no studies of the effects of BrdU on memory or motor behavior, nor have there been studies that systematically varied the age of BrdU treatment. The use of BrdU to identify neurons generated in the adult brain, and the behavioral effects of treatments that retard or enhance this neuronal generation (Gould et al., 1997; Kempermann et al., 1998), make such a study potentially useful to a wide range of investigators.

In the current study rats were given injections of BrdU at one of six different stages of embryonic development (E11, E12, E13, E15, E17, and E21) or on postnatal day (P) 10. The animals were tested behaviorally in adulthood before the brains were harvested and analyzed.

\section{MATERIALS AND METHODS}

There were two experiments. Experiment 1 compared the effect of BrdU (or control) injections on E11, E12, E13, E15, E17, E21, and P10. In adulthood, the animals were tested on a memory task requiring spatial navigation and a fine motor skill task of reaching for food. The general morphology of the brains was then analyzed looking at brain weight, cortical thickness, and relative cerebellar size. Experiment 2 studied only rats with BrdU injections (or control) on E13. The animals were trained in two spatial navigation tasks, a visual detection task, and a visual acuity task.

\section{Experiment 1}

Subjects

Ten pregnant rats, derived from Charles River Long-Evans strains, were randomly chosen to receive injections of BrdU at one of six embryonic stages (seven rats) or to receive no injection (three rats). For the dams that received BrdU, the embryonic dates chosen were E11, E12, E13 ( $n=$ 2), E15, E17, and E21; the rat pups received postnatal injections on P10. On the appropriate day, two injections (spaced $6 \mathrm{hr}$ apart) of $60 \mathrm{mg} / \mathrm{kg}$ $\mathrm{BrdU}$ in $0.007 \mathrm{~N} \mathrm{NaOH}$ or vehicle were given intraperitoneally. All 10 rats were then left to continue their pregnancy naturally. Postnatally, approximately half the rat pups from each litter were given medial frontal lesions at $10 \mathrm{~d}$ of age. (The results of the lesion animals will be reported separately.) The 50 remaining pups from the 10 dams served as the 


\begin{tabular}{lll}
\hline Table 1. Summary of the subjects in Experiment 1 & \\
& $\begin{array}{l}\text { Males } \\
(n=24)\end{array}$ & $\begin{array}{l}\text { Females } \\
(n=26)\end{array}$ \\
Group & 6 & 10 \\
Control & 6 & 5 \\
E11-E13 BrdU $(<$ E13) & 5 & 2 \\
E15 BrdU & 3 & 4 \\
E17 BrdU & 4 & 5 \\
E21 and P10 BrdU & & \\
\hline
\end{tabular}

subjects in this study. Animals that were given BrdU injections from E11 to E13 did not differ on any anatomical or behavioral measure, nor did animals given BrdU injections at E21 and P10. Thus, to simplify the statistical analysis in a study with so many groups, the E11-E13 animals were grouped together, as were the E21 and P10 groups (Table 1).

After weaning, the rats were housed in stainless steel hanging cages where they were maintained on a $12 \mathrm{hr}$ light/dark schedule. Food and water were provided ad libidum except during the reaching task in which they were food-deprived to no less than $85 \%$ of their original body weight. Animals that received BrdU at E11 developed chronic incisor teeth abnormalities and were not food-deprived. Instead, these rats were maintained on a mash form of the normal rat feed throughout the experiment. Testing was conducted during the light cycle in awake and freely moving animals. The body weights of all animals were recorded daily for the first 3 weeks postnatally and then weekly until behavioral testing was completed.

\section{Behavioral methods}

Reaching task. This procedure, developed by Whishaw et al. (1986), was used to assess the skilled forelimb movements of each rat after it was trained to reach for chicken feed pellets in Plexiglas cages $(28 \mathrm{~cm}$ deep $\times$ $20 \mathrm{~cm}$ wide $\times 25 \mathrm{~cm}$ high). The front and floor of each cage were constructed with $2 \mathrm{~mm}$ bars separated from each other by $1 \mathrm{~cm}$, edge to edge. A tray $(5 \mathrm{~cm}$ deep $\times 2 \mathrm{~cm}$ wide $\times 1 \mathrm{~cm}$ high $)$ containing chicken feed pellets was mounted in front of each cage. To obtain food, the rats had to extend the forelimb through the bars, grasp, and retract the food pellet. The food tray was mounted on runners to adjust the distance of the food from the bars. Distance adjustments ensured that each rat could not simply rake the food into the cage. Bars on the floor ensured that if the rat dropped the pellet it would be irretrievable, and the rat would have to reach again. Rats were trained at the task for a maximum of 3 weeks before they were videotaped. During the first week, the rats were grouped in pairs in the reaching cages for $1 \mathrm{hr} / \mathrm{d}$ to allow them to adapt to their new surroundings. The food deprivation schedule commenced during the first week, and each rat was provided with $15 \mathrm{gm}$ of laboratory rodent food daily after the training period. The rats were subsequently trained individually for $1 \mathrm{hr}$ each day during the second week, whereas during the third week this training period was shortened to $5-15 \mathrm{~min} / \mathrm{d}$. Five minutes of continuous reaching activity for each rat was videotaped and scored when the rats were $\sim 5$ months of age. If the rat made a reaching movement (forepaw inserted through the bars, but no food was grasped or it was dropped), it was scored as a "reach," whereas if the rat obtained a piece of food and consumed it, the movement was scored as a "reach" and a "hit." Scoring was achieved by calculating the percentage of hits to total reaches for each animal's preferred forelimb. Left and right paw reaches and hits were recorded separately. Data from the rats in the E11 group were not included in the analyses because they did not learn to reach, which was likely attributable, at least in part, to their teeth abnormalities.

Place task. The method used in this test is similar to that described elsewhere (Sutherland et al., 1983) and is based on the original task described by Morris (1980). The maze is a circular pool $(1.5 \mathrm{~m}$ diameter $\times 0.5 \mathrm{~m}$ deep) with smooth white walls. The pool was filled with $\sim 20^{\circ} \mathrm{C}$ water and mixed with 11 of skim milk powder or just enough to render the water opaque. A clear Plexiglass platform $(11 \times 12 \mathrm{~cm})$ was placed in a constant position inside the pool $12 \mathrm{~cm}$ from the wall. The water level was adjusted so that the platform stood $2 \mathrm{~cm}$ below the surface of the water. The platform was invisible to a viewer outside the pool and to a rat swimming in the water. A trial consisted of placing a rat into the water at one of four locations (north, south, east, or west) around the pool's perimeter. Within a block of four trials each rat started at the four locations in a random sequence, and each rat was tested for four trials per day over 5 consecutive days. If on a particular trial a rat found the platform, it was permitted to remain on it for $10 \mathrm{sec}$. A trial was terminated if the rat failed to find the platform after $90 \mathrm{sec}$. Each rat was returned to its holding cage for $\sim 5 \mathrm{~min}$ before the next trial commenced. The swimming path was traced by the experimenter, and latency to find the platform was recorded. Errors were determined by counting deviations off a direct path from each starting point to the platform. Specifically, the errors were calculated on the traced swim path using a $1.5-\mathrm{cm}-$ wide strip of paper arranged so that it covered the platform and the starting point. Any swim path that was lying outside the direct path was counted as one error. Testing began when rats were 4.5 months old.

\section{Anatomical procedures}

After completion of behavioral testing (at $\sim 7$ months of age), the rats were weighed for a final time, given an overdose of sodium pentobarbital, intracardially perfused with $0.9 \%$ saline, and subsequently fixed with either Lana's fixative (paraformaldehyde and picric acid) or a Golgi-Cox solution. Brain weight and size vary with fixation procedure, so we elected to use the Golgi-Cox-fixed brains for our anatomical measurements. The Lana's fixed brains were used for BrdU immunohistochemistry, which will be reported separately. Golgi-fixed brains were left in solution for $14 \mathrm{~d}$ before being placed in a $30 \%$ sucrose solution for $2 \mathrm{~d}$, cut on a Vibratome at $200 \mu \mathrm{m}$, and developed using the procedure described by Gibb and Kolb (1998). Before sectioning, all brains were photographed from above using a computer imaging program (NIH Image).

Brains were weighed immediately after removal from the skull. The spinal cord was cut even with the caudal edge of the cerebellum.

Cerebellar measurements. Cerebellar measurements were made from the whole-brain photographs. First, the perimeter of the entire brain was traced excluding olfactory bulbs, paraflocculi, and any remaining spinal cord, and then an area measurement was taken. Second, the cerebellum alone was traced, and its area measurement was recorded. The relative size proportion for each brain was calculated by dividing cerebellar area by whole-brain area.

Cortical thickness measurements. Cortical thickness was measured by projecting the Golgi-Cox-stained coronal sections on a Zeiss 2 POL projector set at a magnification of $20 \times$. According to the procedure described elsewhere (Stewart and Kolb, 1988), measurements were taken at three different points (central, medial, and lateral) in each hemisphere at each of five planes. The landmark areas used to determine the correct planes were as follows: Plane 1, first plane with caudate-putamen visible; Plane 2, anterior commissure; Plane 3, first hippocampal section; Plane 4, posterior commissure; and Plane 5, most posterior hippocampal section. A plastic centimeter ruler was used to measure from the edge of the white matter to the outer edge of the cortex. An average for each plane and for each rat was calculated and used for statistical comparisons. Because of the small number of female brains fixed with Golgi-Cox, measurements of cortical thickness are reported only for male brains.

\section{Experiment 2}

Because spatial learning and memory deficits can have so many different causes, the goal of this experiment was to examine the basis of the impairment in the place task. The rats were given tests of reference memory, working memory, cue learning, and visual ability. Two pregnant rats received either BrdU or vehicle on E13 as in Experiment 1. In total, there were nine control rats (five females, four males) and nine BrdU-injected rats (five females, four males). The animals were housed and cared for as in Experiment 1. At the end of the experiment the animals were overdosed with Euthanol and perfused with $0.9 \%$ saline and $10 \%$ formalin.

\section{Spatial learning tasks}

Place learning: reference memory. This task is identical to the water task in Experiment 1 except that the animals were given only one trial per day for a total of 10 consecutive days to reduce the motor demands. After the place learning trials were completed, the platform was removed, and the animals were allowed to swim for $60 \mathrm{sec}$. The percentage of time the animals spent in each quadrant was recorded.

Matching to place: working memory. After the rats completed the probe trial, the platform was placed in a new location (northeast quadrant) on each of $10 \mathrm{~d}$. The rats were given two trials each day. Each rat was placed in the pool facing the wall at a cardinal compass point opposite the location of the platform. The rats were allowed to swim until they found 
this platform. Once they found the platform, they remained there for 10 sec. Then they were returned to their starting point to begin their second trial or returned to their home cage for $2 \mathrm{hr}$ before they were given a second trial. A $10 \mathrm{sec}$ delay was used on five trials, and a $2 \mathrm{hr}$ delay was used on another five trials.

\section{Cue and visual detection task}

The goal of this task was to teach rats to swim to a target that identified the location of a hidden platform in the same water tank used for the spatial learning tasks. The targets varied in size, thus providing a measure of the rats' ability to visually detect objects of different sizes.

Targets. Seven targets were constructed to sit on top of the submerged platform. The targets were cylindrical in shape and protruded $5 \mathrm{~cm}$ above the water. The 50 and $70 \mathrm{~mm}$ (diameter) targets were constructed of ABS plastic, whereas the $3,6,15$, and $25 \mathrm{~mm}$ targets were constructed of wooden doweling. The $1 \mathrm{~mm}$ target was constructed of a thin metal rod and was affixed to the platform with modeling clay. The vertical surface of each target was painted black to provide maximum contrast with the white background of the pool walls when viewed from the water surface, whereas the horizontal surfaces (top) of the targets were painted white to avoid interference with the movement-tracking computer.

Video tracking and analysis. The animals' movements in the pool were detected by a video motion tracking system (San Diego Instruments). The time taken to locate the platform, the total distance traveled in finding the platform, and the distance from the animals' final turn to the target were recorded. The values for final turn to target distance were determined to be the distance from which the animal could see the target.

Procedure. The platform was placed in the pool at random locations for each trial for the duration of the experiment. During the first $3 \mathrm{~d}$, the 70 $\mathrm{mm}$ target was placed on top of the platform. The rats were placed gently in the pool facing the wall at the east cardinal compass point; they received six trials per day. After this time the rats were capable of swimming directly to the platform. Using the same procedure, the rats were then tested on the various smaller target sizes in random order, including control trials in which no target was available, only a submerged platform. The rats were tested over the course of $6 \mathrm{~d}$ on all targets.

\section{Visual acuity task}

Visual acuity is defined as the ability to visually distinguish fine details. It can be measured by determining the ability of subjects to distinguish between lines of different spatial frequency. In the current task, which was devised by Prusky and Douglas (1998), the rats were trained to distinguish between a screen displaying a low spatial frequency grating and a screen displaying gray of the same mean luminance. If animals could detect the fine lines, they could differentiate between the two discriminants. Once the lines became too fine for them to differentiate, both gratings would appear gray, and they would be unable to respond differentially to them.

Apparatus. The testing apparatus consisted of a trapezoid-shaped swimming pool with a length of $140 \mathrm{~cm}$ and a width of $80 \mathrm{~cm}$ at the wide end and $26 \mathrm{~cm}$ at the narrow end. The pool walls were $35 \mathrm{~cm}$ high, and the pool was filled to a depth of $\sim 14 \mathrm{~cm}$. The pool was constructed of transparent plastic with critical areas painted with nonreflective paint to prevent reflection. At the wide end of the pool, two 17 inch monitors presented spatial gratings. The display on the screen was controlled by Vista software. A barrier $46 \mathrm{~cm}$ long and $70 \mathrm{~cm}$ high was in the center of the pool to force subjects to make a binary decision. A platform $12 \mathrm{~cm}$ high and $38 \mathrm{~cm}$ wide was placed below one monitor.

Procedure. Subjects were trained to swim toward the screen with a grating on it using a shaping procedure. Once animals were swimming the length of the pool to escape via the platform, they were given a series of trials with a grating that corresponded to a spatial frequency of $\sim 0.1$ cycles/degree. At this low spatial frequency, the animals can easily make accurate decisions to swim to the correct side of the pool. Once the animals were making correct decisions at least $70 \%$ of the time, the frequency of the grating was increased, and the animals were tested on a staircase method. To make certain that the animals were making correct choices, animals had to either correctly respond to four trials consecutively or correctly respond to 7 of 10 trials before they were tested on the next higher grating. The proportion of correct trials at each spatial frequency for each animal was calculated.

\section{RESULTS}

\section{General body morphology}

Rats that received BrdU earlier than E21 had coat patterns that deviated from the normal Long-Evans hooded characteristics. Normally, these rats have white bodies with black heads and a thin (and variable) black stripe along the dorsal midline. BrdU-treated rats maintained these basic features, but in addition they had black, Dalmatian-like spots indicating interference with the normal melanocyte distribution during development. The extent and size of the spots varied across the different BrdU groups, and Figure 1 shows the ventral coat of rats that are characteristic of each group. E11 rats had only a few large spots, whereas E12 and E13 rats had many large spots. The spots on the E15 and E17 rats were smaller than the spots in the other groups. The E21 rat coats appeared to be unaffected.

Some rats in the E11 BrdU group had further morphological abnormalities. Four of the seven rats in this litter had permanently kinked tails that were bent in two or three different areas (Fig. 2). Three out of the seven rats had polydactyly in at least one paw, and all of the rats in this litter had abnormal incisor growth. Both top and bottom incisors grew either crooked or curved inward into the opposite jaw. In either situation, the rats could not maintain their teeth by gnawing, and they needed to be clipped weekly by the animal care technician. Even with this care, the postweaning mortality of these rats was high, and by the time of behavioral testing, only two of the six original animals survived.

Finally, rats that received BrdU injections on P10 lost their body fur. This fur regrew, and by the time of weaning the animals were indistinguishable from untreated rats.

\section{Behavioral results: Experiment 1}

Overall, BrdU rats were noticeably easier to handle and test in behavioral tasks than the normal rats, who were handled equal amounts of time. The prenatal BrdU rats seldom struggled or scratched when being handled, even in relatively stressful situations such as the water task training sessions. There were no sex differences on any task, so the data from both sexes were combined.

\section{Reaching task}

Normal control rats were successful in grasping and eating the food pellets $\sim 60 \%$ of the time. Rats exposed to BrdU on E17P10 performed like control animals, but those rats exposed to BrdU on E15 or earlier were markedly impaired. The top portion of Figure 3 summarizes the reaching accuracy as a percentage for the different groups, and it is apparent that the E15 group is very poor indeed. The bottom portion of Figure 3 graphs the percentage of animals that performed below 1 SD from control mean. The E11 rats were not included in the graphs or the statistical analysis, because none learned to reach. ANOVA on the reaching accuracy scores revealed a significant main effect $\left(F_{(4,43)}=5.3\right.$; $p<0.002)$. Post hoc tests (Fisher's least significant difference) found that only the E15 group differed significantly from all other groups. No other differences were significant.

\section{Place task}

When initially placed into the water, all rats swam around the outer perimeter of the pool. Control animals quickly abandoned this pattern and traversed a wide area until they incidentally bumped into the platform. From this point, control rats quickly learned to relocate and swim directly to the platform from any of the four start positions. Performance improved until the third 

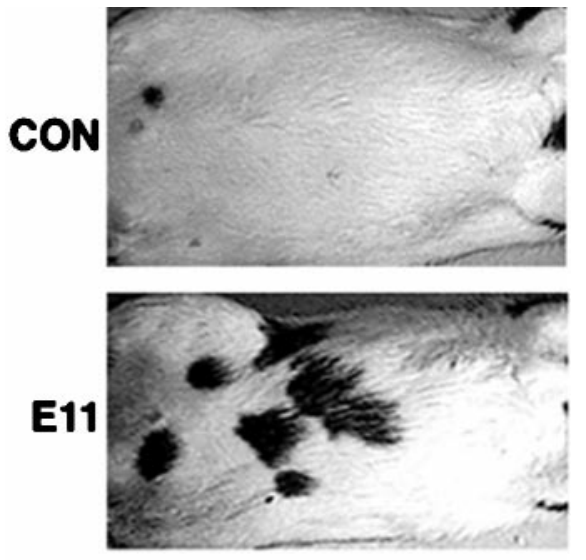

E12

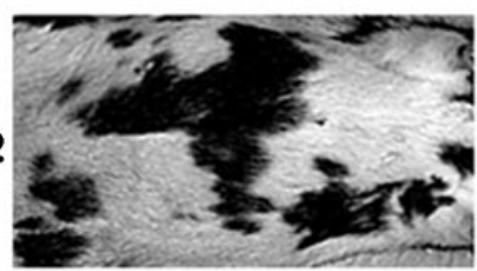

E13

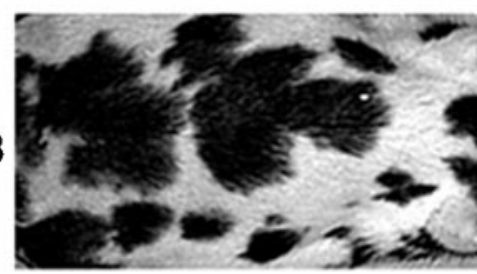

E15
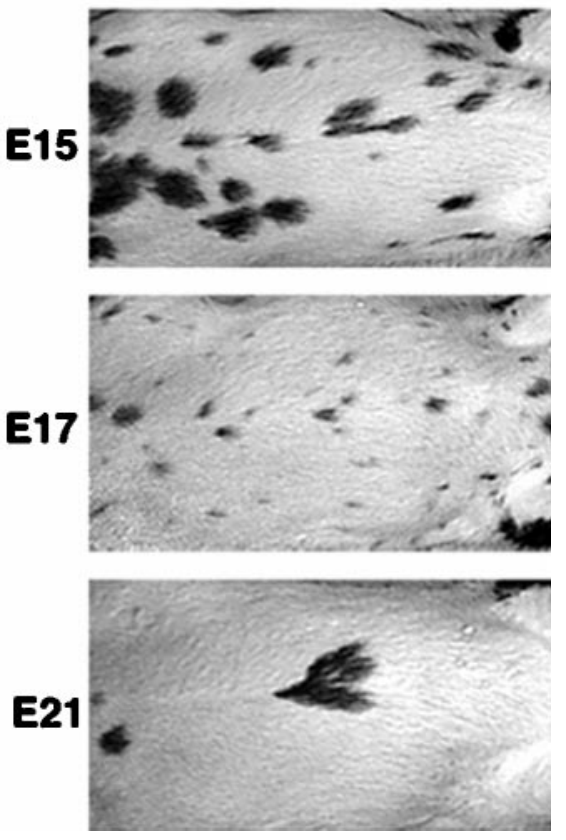

Figure 1. Photographs of the ventrum of male rats exposed to vehicle $(C O N)$ or BrdU at different embryonic ages. The head is to the right. Injections at E11-E13 produced large spots; injections at E15-E17 produced progressively smaller spots. Injections at E21 or P10 had no effect on coat pattern.

trial block in which it reached asymptote at $\sim 5 \mathrm{sec} /$ trial. The average total escape time across all trial blocks for the control rats was $73 \mathrm{sec}$ (Fig. 4). Collectively, rats exposed to BrdU on E17 and earlier performed more poorly on the water task. These animals were less likely to adopt a swim pattern that would quickly allow them to find the platform. Additionally, it took these rats longer to relocate the platform after successfully finding it in previous trials. As in the reaching task, however, exposure to BrdU at E21 or P10 did not influence water task performance. One additional behavior is worthy of note. Rats normally swim with their forepaws tucked under their chins. The E11 rats did not inhibit their forelimbs, however, because they swam "dog paddle" style. We have observed this behavior in decorticate rats (Kolb and Whishaw, 1981).

ANOVA on total latency revealed a significant main effect $\left(F_{(4,45)}=10.4 ; p<0.0001\right)$. Post hoc tests showed that the $<\mathrm{E} 13$ group differed from control, and the E17 group differed from all other groups.

\section{Error analysis}

Figure 5 shows the mean swim path errors. On the early trials the animals did not know the route to the platform and thus made an error on virtually every trial. Over trials, the animals in all groups except the $<$ E13 group showed a significant drop in errors, indicating that the animals had learned the location of the platform. The $<$ E13 group showed no improvement, however, which indicates that although they became more proficient at the task, they likely had learned a strategy to find the hidden platform but did not learn to swim directly to the platform. Curiously, the swim paths of the $>$ E21 group were more accurate than those of the control group.

ANOVA on heading errors showed a significant main effect $\left(F_{(4,45)}=4.9 ; p<0.002\right)$. Post hoc tests showed that the $<\mathrm{E} 13$ and E17 groups differed from control.

Unlike results found elsewhere (Kolb and Cioe, 1996; Kolb et al., 1996), sex was not a factor in any of the measurements made across any of the groups tested in the water task. The difference between studies, however, is that the rats in this experiment swam four trial blocks per day instead of eight. We have found that the four-trial procedure leads to faster learning, so it appears that sex differences emerge only when the task is made more difficult.

\section{Behavioral results: Experiment 2}

Overall, the BrdU-treated animals were impaired at the spatial learning tasks and had reduced visual acuity.

\section{Spatial learning tasks}

Place learning. As in Experiment 1, the BrdU-treated rats were impaired relative to the control animals and did not actually learn the location of the hidden platform (Figs. 6, 7). The computerized analysis of swimming performance indicated that although the BrdU-treated rats had longer latencies and swam further than control rats, their swim speed was identical (Fig. 6).

ANOVA on latency with group and trial as factors showed significant main effects of both group $\left(F_{(1,16)}=6.7 ; p<0.02\right)$ and trial $\left(F_{(9,144)}=6.9 ; p<0.0001\right)$ but not the interaction $\left(F_{(9,144)}=\right.$ $0.9 ; p=0.51)$. The results for swim distance were virtually identical: group $\left(F_{(1,16)}=4.8 ; p<0.04\right)$, trial $\left(F_{(9,144)}=6.0 ; p<\right.$ $0.001)$, group $\times \operatorname{trial}\left(F_{(9,144)}=10.0 ; p<0.001\right)$. There were no significant effects on swim speed, however $(p>0.2)$.

ANOVA on the probe trial showed no effect of treatment $\left(F_{(1,16)}=0.6 ; p=0.46\right)$, but there was a significant effect of swim quadrant $\left(F_{(3,48)}=18.5 ; p<0.0001\right)$, as well as an interaction $\left(F_{(3,48)}=11.1 ; p<0.0001\right)$.

Matching to place. The BrdU-treated animals were impaired at learning the matching-to-place task, with both the $10 \mathrm{sec}$ and $2 \mathrm{hr}$ 

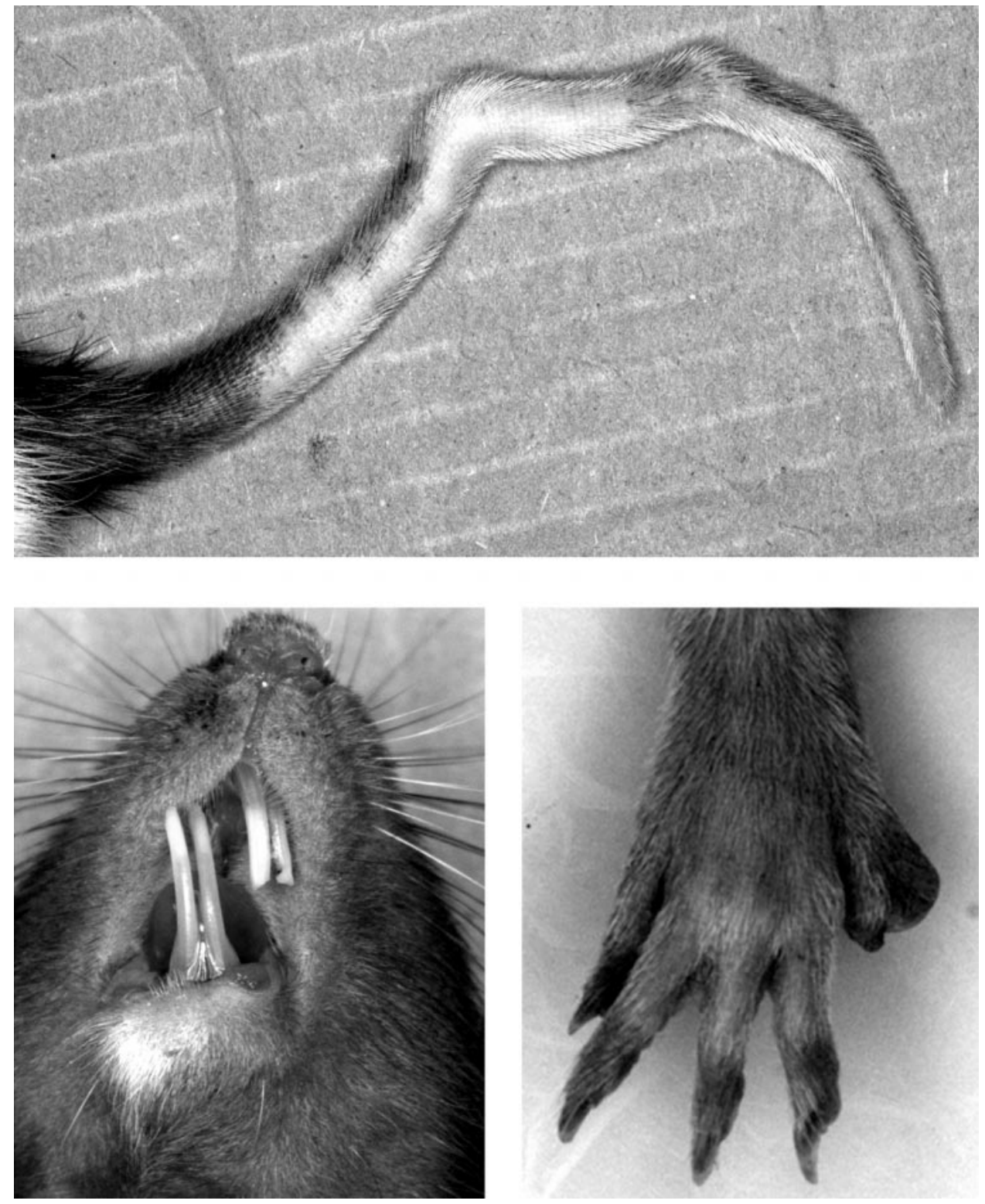

Figure 2. Photograph of physical abnormalities in teeth, digits, and tails of rats with E11 BrdU injections. These abnormalities were not present in any animals with later injections. delays between trials. A two-way ANOVA with group and delay interval as factors found significant effects on both latency to find the platform on trial $2\left(F_{(1,16)}=7.8 ; p<0.01\right)$ and swim distance on trial $2\left(F_{(1,16)}=9.2 ; p<0.01\right)$. There was no effect of interval for either measure nor were there interactions $(p>0.4)$.

Visual detection task. The animals learned the task quickly and learned to swim directly to the target. There was a group difference, however, because the BrdU-treated animals were slower to find the platform at all target sizes, except the second largest one (Fig. 8). ANOVA on latency and swim distance revealed significant main effects of group $\left(F_{(1,16)}=18.8 ; p<0.001\right.$; $\left.F_{(1,16)}=14.0 ; p<0.002\right)$ and target size $\left(F_{(5,80)}=36.8 ; p<\right.$ $0.0001 ;\left(F_{(5,80)}=27.7 ; p<0.0001\right)$, but the interaction was not significant $\left(F_{(5,80)}=2.0 ; p=0.09 ;\left(F_{(5,80)}=1.9 ; p=0.10\right)\right.$.

Visual acuity task. The mean visual acuity of the control rats was 0.85 cycles/degree, whereas that of the BrdU-treated rats was 0.69 cycles/degree. Figure 9 shows that although the two groups performed equally well at low spatial frequencies, the performance of the BrdU-treated rats dropped off rapidly after 0.6 cycles/degree, whereas many of the control animals continued to perform very well up to $\sim 9$ cycles/degree. ANOVA with group and spatial frequency as factors showed a nonsignificant group effect $\left(F_{(1,3)}=7.1 ; p=0.08\right)$, but the spatial frequency $\left(F_{(23,69)}=\right.$ $10.7 ; p<0.0001)$ and interaction effects $\left(F_{(23,69)}=2.8 ; p<0.001\right)$ were significant. This reflects the similar performance of the two groups at the easier spatial frequencies but better performance of the control animals at the higher frequencies.

\section{Anatomical results}

Body weight

BrdU exposure on E15 and earlier led to decreased body weights, which can be seen for the data for day 100 (Table 2). In contrast, BrdU exposure on E21 or P10 did not decrease body weight.

An ANOVA with treatment and sex as factors showed a significant main effect of treatment $\left(F_{(4,42)}=7.6 ; p=0.0001\right)$ and of $\operatorname{sex}\left(F_{(1,42)}=180.6 ; p<0.0001\right)$, but the interaction was not significant $\left(F_{(4,42)}=0.95 ; p=0.45\right)$. Post hoc tests revealed that only the E15 group differed from control. 

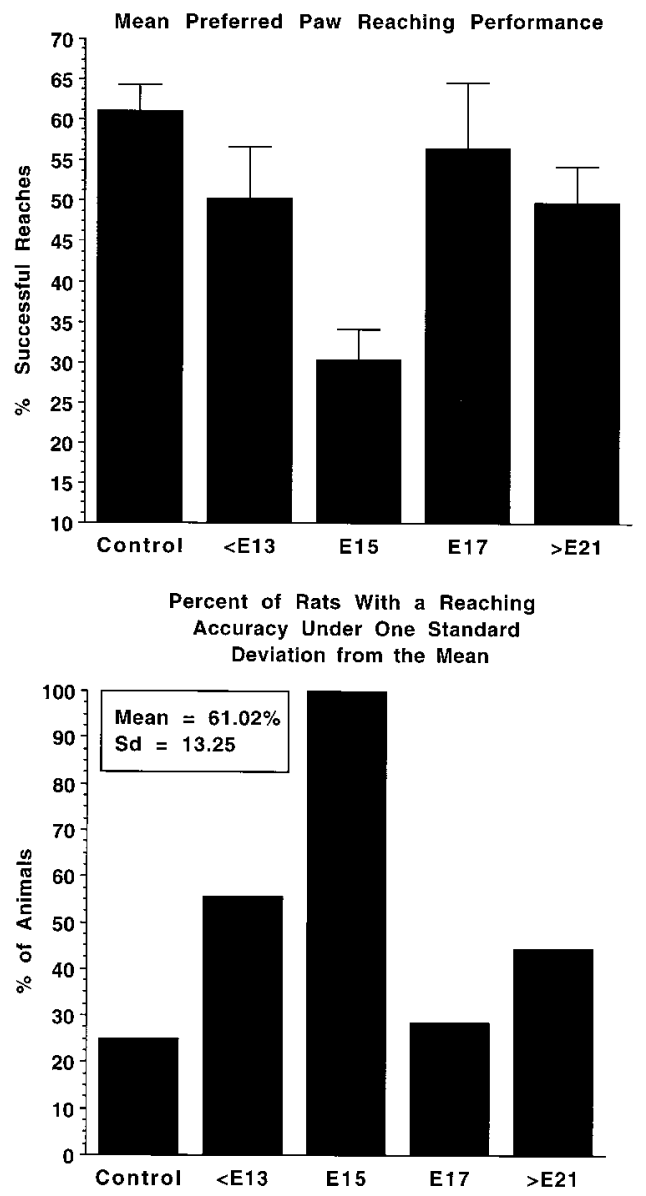

Figure 3. Summary of skilled forelimb reaching. Rats with E15 BrdU injections were severely impaired at the task. The top panel shows reaching accuracy. The bottom shows the percentage of rats in each group that performed worse than $1 \mathrm{SD}$ below control mean.

\section{Brain weight}

Rats with BrdU exposure on E15 or earlier had smaller brains than rats in the other groups (Table 3; Fig. 10). ANOVA was significant $\left(F_{(4,18)}=10.8 ; p<0.0001\right)$. The $<$ E13 and E15 groups differed from control.

\section{Cerebellar measurements}

During examination of the rat brains, it became apparent that the cerebellums of the $<$ E13 BrdU-exposure rats were abnormally small (Fig. 10). In particular, the vermis was concave, although the lateral lobes were obviously small as well. Figure 11 shows the relative cerebellar area to whole-brain area as a percentage. Control rat cerebellums constituted $\sim 23 \%$ of the total surface area of the brain, whereas the cerebellums exposed to BrdU before E13 constituted only 19.5\%. Cerebellums that were exposed to BrdU from E15-E21 were similar to the controls, but the P10 group was smaller. ANOVA on cerebellum to whole brain ratio was significant $\left(F_{(4,45)}=16.1 ; p<0.0001\right)$. Post hoc analysis determined that the $<$ E13 and P10 groups had significantly smaller cerebellums.

\section{Cortical thickness}

The overall result was that brains exposed to BrdU on E17 and earlier had thinner cortices than control or $>$ E21 BrdU-exposed brains (Fig. 12). The effect on cortical thickness was general
Total Latency

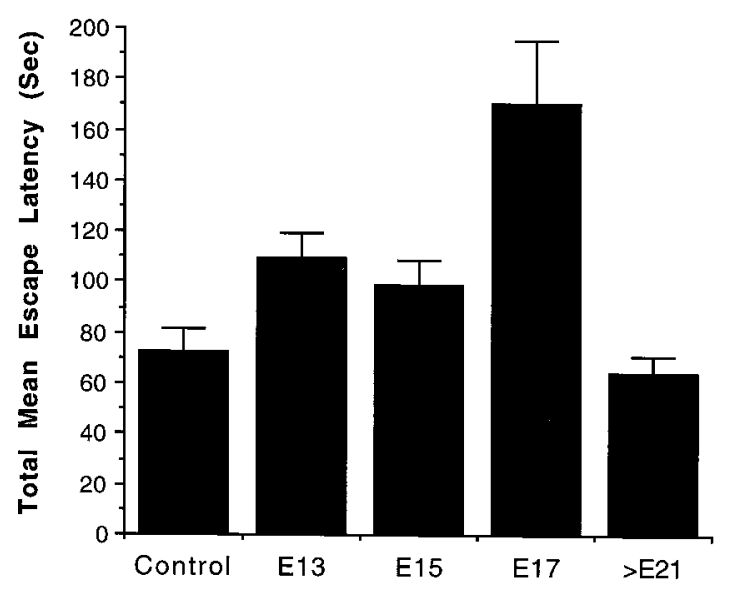

Trial Block Latency

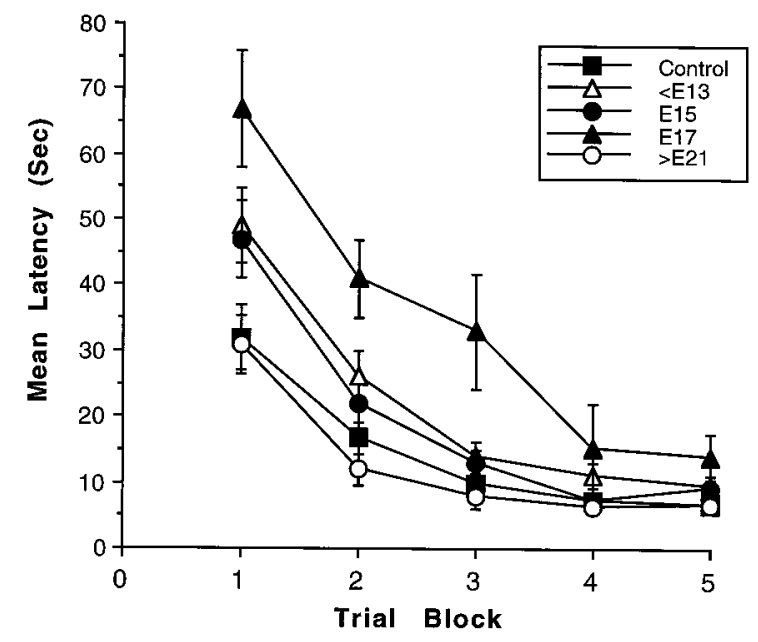

Figure 4. Summary of latency performance on the Morris water task. The top panel shows the mean total latency summed across all 20 trials. The bottom shows performance on each day of training.

across the cortex, except for the frontal measure, where there were no differences.

ANOVA on overall cortical thickness was significant $\left(F_{(4,29)}=\right.$ 7.2; $p<0.001)$. Post hoc tests showed that the $<\mathrm{E} 13$, E15, and E17 groups differed from the control and $>$ P21 groups, which did not differ.

\section{DISCUSSION}

Our results demonstrate that embryonic exposure to bromodeoxyuridine, especially on E17 or earlier, affects body and brain morphology and behavior in rats. The teratological effects of BrdU likely vary with the day of exposure and the particular developmental processes that are in progress at that time. It would be difficult to identify the precise differences between different embryological days, however, because teratological effects at any age of development might be expected to have widespread sequelae. Nevertheless, it is possible to make general statements about early and later effects of BrdU exposure. Rats exposed to BrdU at E17 or earlier had reduced body weights throughout the experiment, which is consistent with results re- 


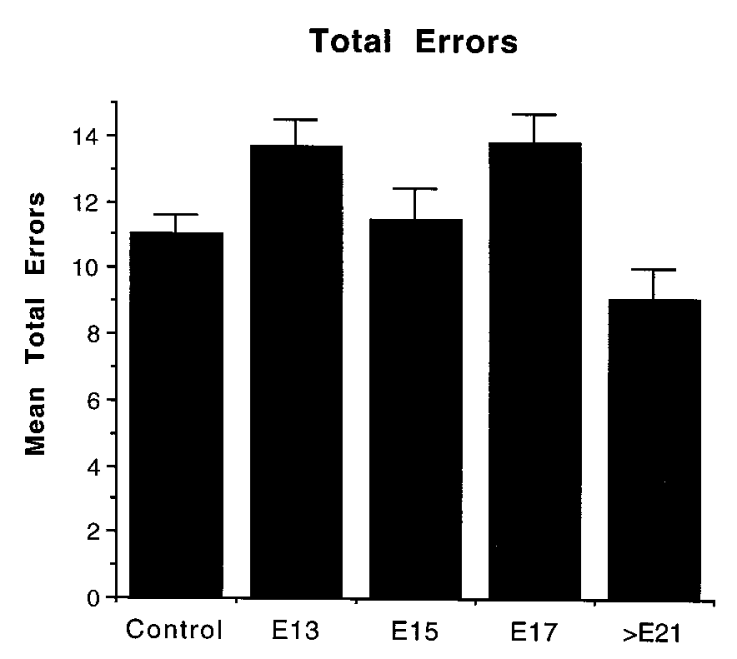

\section{Trial Block Errors}

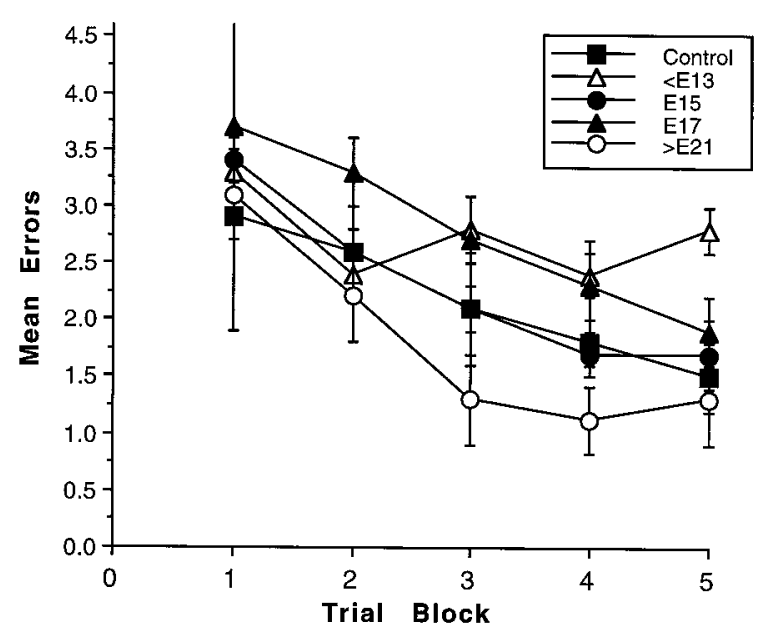

Figure 5. Summary of error scores on the Morris task. The top panel shows the mean total errors summed across all 20 trials. The bottom shows performance on each day of training. The maximum number of errors per day is four.

ported elsewhere (Yu, 1976; Nagao et al., 1997). A striking alteration in phenotype was the abnormal spotting patterns on the rats' fur. The pattern of Dalmatian-like fur varied across the exposure dates, suggesting that BrdU alters any derivatives formed from the primary germ layers. Because fur pattern is most affected by exposure to BrdU at E12-E15, it appears that the regulation of melanocyte distribution is critical during this time period. Although we are unaware of other reports of alterations to fur coat patterns, there are reports of pigmentation alterations after exposure to BrdU in cultured chick retinal cells (Zimmerman et al., 1974; Garcia et al., 1979) and mouse melanoma cells (Wrathall et al., 1973).

Rats exposed to BrdU at E11 developed abnormal incisor growth, and many also developed kinked tails and polydactyly of at least one limb. The incisors of these rats grew crooked either laterally or inward into the mouth and gums of the opposite jaw. If left unchecked, the abnormal incisors would cause serious problems for these rats. Polydactyly has been found previously to occur in mice that were subjected to repeated doses of $\mathrm{BrdU}$
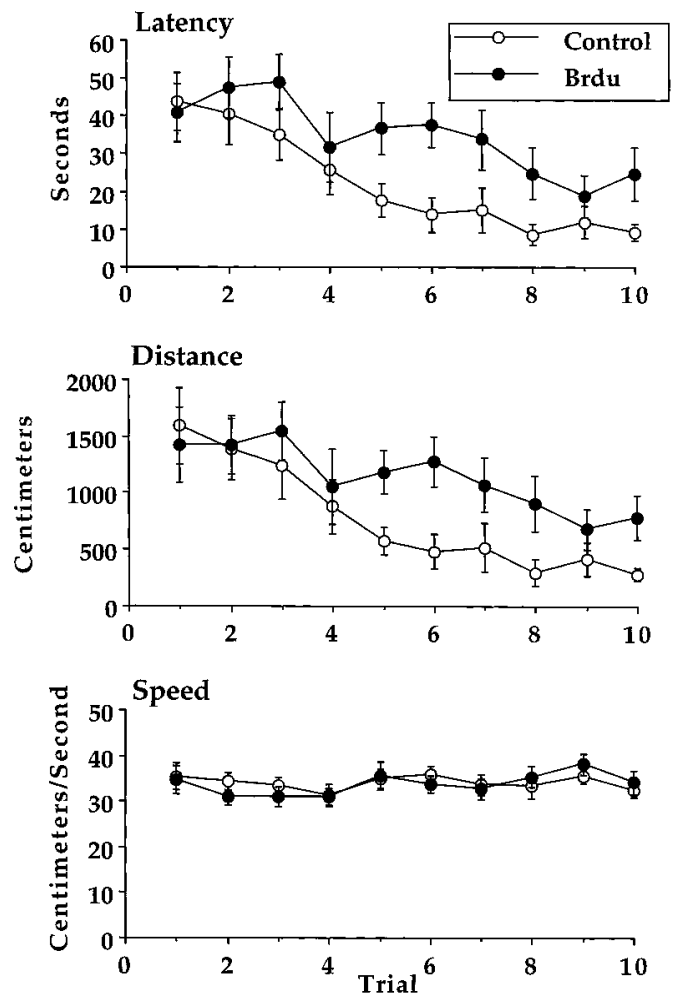

Figure 6. Summary of performance in the Morris task in Experiment 2. There was only one trial per day. The top panel summarizes time to find the hidden platform, the middle summarizes the distance swum to find the platform, and the bottom summarizes the swim speed. Rats with E13 $\mathrm{BrdU}$ injections took longer to find the platform and swam further but swam at the same speed as control rats.

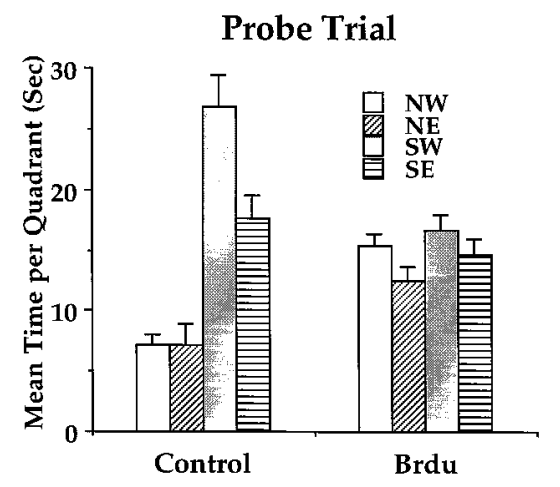

Figure 7. Performance on the probe trial in the Morris task in Experiment 2. The platform had been located in the southwest quadrant. Control animals preferentially searched in this quadrant for the platform. The BrdU-injected rats did not.

embryonically at days 6 through 9 (DiPaolo, 1964). It is interesting to note that the rats exposed to BrdU at E11 have some similarities to the mutant $(\mathrm{Cd})$ crooked-tail mouse. The common features of the heterozygote mutant mice are kinked tails and exencephaly, whereas the homozygotes have a runted phenotype, thinner cortex, and reduced dendritic arborization (Carter et al., 1997). Preliminary results indicate that cortical pyramidal dendritic arborization in the E11 rats is stunted (Kolb et al., 1997), and neural tube deficits and dwarfism were found when early hamster embryos were subjected to BrdU (Ruffolo and Ferm, 1965). The comparison between the effects of BrdU exposure and 

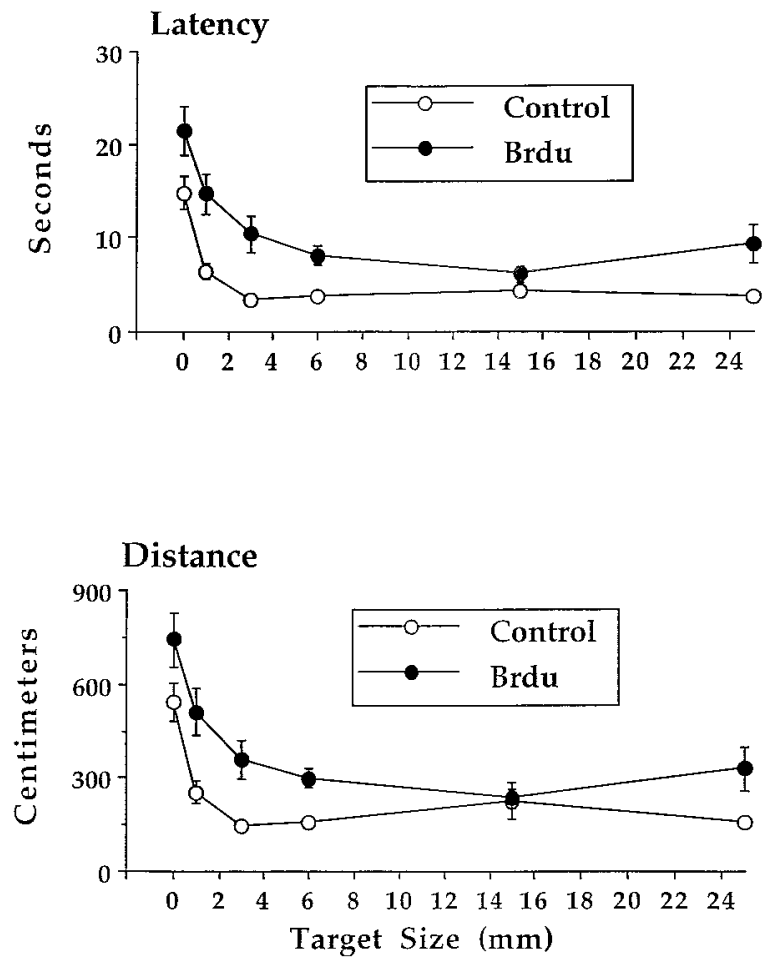

Figure 8. Summary of performance in the visual detection task. The BrdU-treated rats were slower to locate the platform, which was indicated by a target of different sizes, than the control animals.

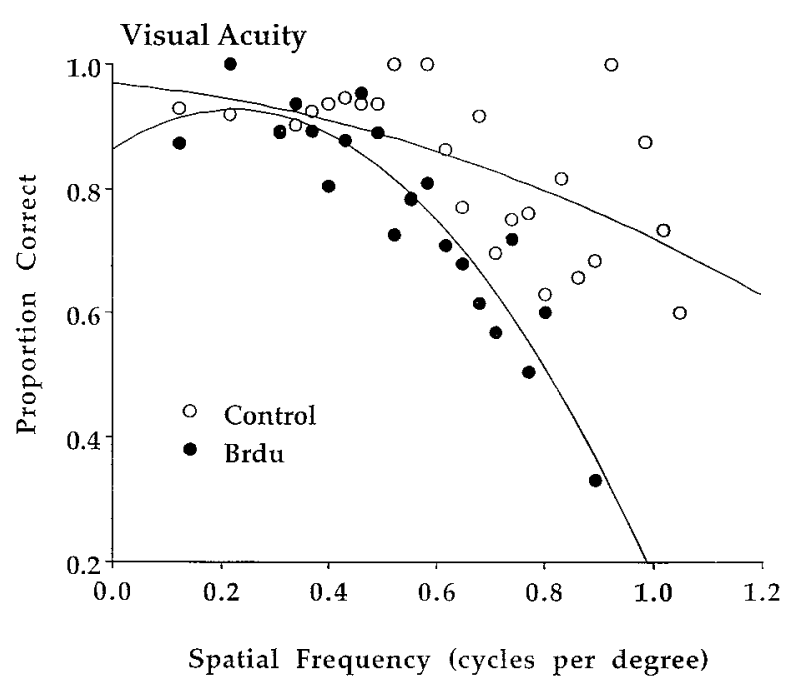

Figure 9. Summary of the performance of the rats on the visual acuity task. The performance of the BrdU rats dropped off to chance levels around 0.7 cycles/degree, whereas the performance of the control rats was considerably better.

the mutant strain of mice suggests that early BrdU exposure can result in an identifiable mutation.

BrdU treatments had wide-ranging effects on motor and cognitive behavior because rats were impaired in skilled motor performance and cognitive tasks. Although the deficits were age-related, it is unlikely that they are specific. For example, rats receiving BrdU treatments were impaired on reference memory and working memory as well as cue detection tasks and additionally had reduced visual acuity. Although it is possible that motor,

\begin{tabular}{lll}
\hline Table 2. Body weights at 100 & $\mathbf{d}$ of age \\
& $\begin{array}{l}\text { Males } \\
(n=24)\end{array}$ & $\begin{array}{l}\text { Females } \\
(n=26)\end{array}$ \\
Group & $434 \pm 6$ & $278 \pm 7$ \\
\hline Control & $381 \pm 22^{*}$ & $266 \pm 8$ \\
E11-E13 BrdU $(<$ E13) & $334 \pm 11^{*}$ & $212 \pm 2^{*}$ \\
E15 BrdU & $430 \pm 3$ & $265 \pm 13$ \\
E17 BrdU & $430 \pm 33$ & $285 \pm 17$ \\
E21 and P10 BrdU &
\end{tabular}

*Differs significantly from control $(p<0.05)$.

\section{Table 3. Summary of brain weights}

\begin{tabular}{ll} 
Group & Weight \\
\hline Control & $2.14 \pm 0.04$ \\
E11-E13 BrdU $(<$ E13) & $1.89 \pm 0.04^{*}$ \\
E15 BrdU & $1.92 \pm 0.03^{*}$ \\
E17 BrdU & $2.01 \pm 0.01$ \\
E21 and P10 BrdU & $2.19 \pm 0.07$
\end{tabular}

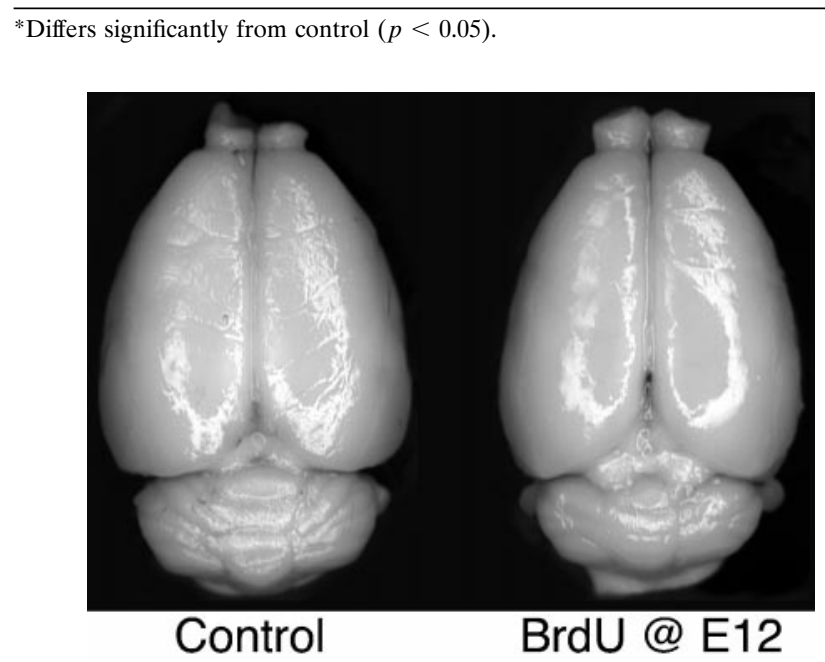

Figure 10. Photographs of a control brain and an E12 BrdU-treated brain. The cerebral and cerebellar hemispheres of the BrdU brain are visibly smaller.

learning, and perceptual deficits are independent, the possibility that motor and perceptual deficits contribute to the cognitive deficits cannot be excluded. Nevertheless, it likely that the behavioral deficits are a consequence of CNS changes. The exposure to BrdU early in embryonic development (on or before E17) led to a $10 \%$ decrease in brain weights and $5 \%$ thinner cerebral cortices. In addition, the cerebellums were smaller in relation to wholebrain size and appeared truncated or concave with exposure to BrdU on or before E15. In some regards, brains exposed to BrdU early in development resemble brains that have received a postnatal lesion. Indeed, Kolb (1987) has shown that neonatal P10 cortical lesions result in brains that weigh $\sim 20 \%$ less than control brains, and the remaining cortex in these animals was $\sim 10-20 \%$ thinner. Similarly, DiPaolo (1964) referred to abnormalities produced by BrdU as a metabolic or biochemical lesion.

The effect of BrdU on cerebellar size has been reported elsewhere (Yu, 1976; Bannigan and Langman, 1979). Yu (1976) suggested that incorporation of BrdU into mitotically active cells caused inhibition of cell formation and may have destroyed the stem cell population of the external granular layer. Neurogenesis 
Relative Cerebellar Size

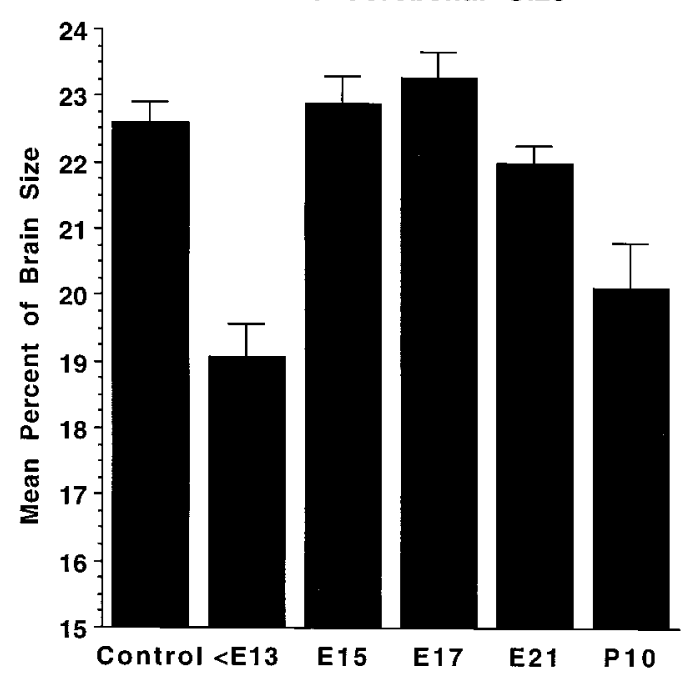

Figure 11. Summary of the ratio of the cerebellar area (from dorsal photographs) to the total cerebral + cerebellar area. The rats with injections at E13 or earlier or at P10 have relatively smaller cerebellums.

and cell proliferation in the cerebellum continue postnatally except for Purkinje cells, which complete their final mitosis just before birth. The result that cerebellar size decreased after exposure to BrdU on E11 through E15 could suggest alterations to the stem cell population early in development. Subsequently, during a period of rapid cell proliferation, either the daughter cells are unable to differentiate or the cell cycle has been altered sufficiently to prevent normal development of the cerebellum. The latter would agree with results found by Bannigan and Langman (1979), who exposed mice fetuses to BrdU at E12 when Purkinje cells are being formed. They found $15 \%$ fewer Purkinje cells in the postnatal cerebellum that was exposed to BrdU embryonically. Bannigan and Langman (1979) concluded that cells that had incorporated the BrdU into the DNA were able to migrate from the proliferation zone to the periphery and differentiate into normal cells. The decrease in cell population was a result of cell death and a longer cell cycle time. However, Yu (1979) found that postnatal exposure to BrdU led to a decreased molecular layer width in the cerebellum, and that the Purkinje cells had abnormally long primary dendrites. Yu concluded that a lengthened cell cycle time reduced the regenerative capabilities of the stem cell population in the external granular layer. Finally, we should note that our observation of reduced cerebellar size was post hoc, because we examined photographs of the brains. Thus, because the cerebellar tissue had not been kept for anatomical analysis, we were able to make only a two-dimensional areal measurement. It is quite likely that a volumetric measurement would have yielded a larger effect on cerebellar development.

Alterations to the stem cell population or a lengthened cell cycle time could provide explanations as to why the cortices of the brains exposed to BrdU are thinner than controls. Webster et al. (1973) found that embryonic exposure to BrdU at E15 prolonged the duration of mitosis in neocortical cells, but the mitosis itself was not blocked. When the cells were looked at later in development, they noticed that many cells became darkly nucleated in the neuroepithelial zone and beyond. They noted that these cells gradually disappeared, probably becoming inviable and ultimately causing a reduction in cortical cell number. Cells that have
Mean Cortical Thickness

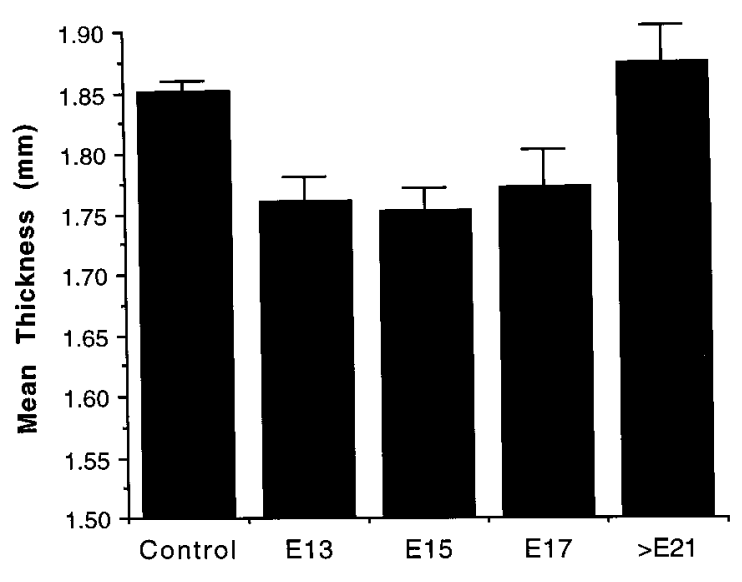

Thickness by Plane of Section

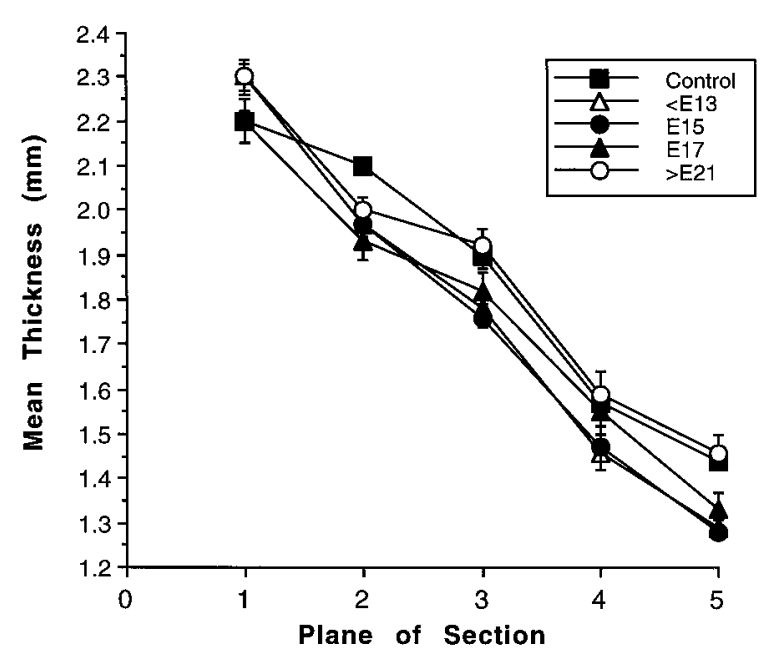

Figure 12. Summary of cerebral cortical thickness. The top panel shows the mean overall thickness, and the bottom panel shows the mean thickness at each of five planes. Plane 1 is the most anterior. Rats with BrdU injections between E11 and E17 have thinner cerebral cortex than the control and $>$ E21 rats.

incorporated BrdU into the DNA may not be able to sustain adequate regulation or produce appropriate factors needed to survive and may undergo a process similar to apoptosis. Because different cell populations that are destined for different layers are generated at different embryonic ages, we would anticipate that different cortical layers might be thin in the different groups. This could not be determined from Golgi-stained sections but would be a useful measure in future studies.

The age-dependent results of BrdU treatment lead us to three conclusions. First, because we have used doses of BrdU that typically are used in developmental studies as well as studies in adult animals, one should be aware that BrdU significantly alters both brain and behavior. The finding that embryonic exposure to BrdU alters normal development is not a novel finding, but previous research has not recognized the severe and wide-ranging morphological and behavioral effects of the treatment. Second, the age-dependent changes in both morphology and behavior imply that BrdU is altering the nervous system differently at different ages and that the effects of BrdU are not limited to the 
embryonic period. Third, the effects of BrdU treatment at different ages are not completely selective, because motor, cognitive, and perceptual deficits could all be obtained at a single time point. The findings of this study should be cautionary for those who use BrdU as a mitogenic marker and conduct behavioral studies (Kempermann et al., 1998). It may be that BrdU alters the very phenomena that investigators are studying. Nevertheless, BrdU may be useful for the study and treatment of tetragenetic effects on morphology and behavior.

\section{REFERENCES}

Agnish ND, Kochhar DM (1976a) Direct exposure of postimplantation mouse embryos to 5-bromodeoxyuridine in vitro and its effect on subsequent chondrogenesis in the limbs. J Embryol Exp Morphol 36:623-638.

Agnish ND, Kochhar DM (1976b) Direct exposure of mouse embryonic limb-buds to 5-bromodeoxyuridine in vitro and its effect on chondrogenesis: increasing resistance to the analog at successive stages of development. J Embryol Exp Morphol 36:639-652.

Bannigan J, Langman J (1979) The cellular effect of 5-bromodeoxyuridine on the mammalian embryo. J Embryol Exp Morphol 50:123-135.

Barasch JM, Bressler RS (1977) The effect of 5-bromodeoxyuridine on the postnatal development of the rat testis. J Exp Zool 200:1-8.

Bick MD, Davidson RL (1974) Total substitution of bromodeoxyuridine for thymidine in the DNA of bromodeoxyuridine-dependent cell line. Proc Natl Acad Sci USA 71:2082-2086.

Carter ML, Forster C, Bhide PG, Caviness VS, Ross ME (1997) The crooked tail $(\mathrm{Cd})$ mutation interferes with neuronal positioning in cerebral cortex. Soc Neurosci Abstr 23:871.

DiPaolo JA (1964) Polydactylism in the offspring of mice injected with 5-bromodeoxyuridine. Science 145:501-503.

Dolbeare F (1994) Bromodeoxyuridine: a diagnostic tool in biology and medicine. Part I: Historical perspectives, histochemical methods and cell kinetics. Histochem J 27:339-369.

Dribin LB, Jacobson M (1978) Effects of 5-bromodeoxyuridine on development of Mauthner's neuron and neural retina of Xenopus laevis embryos. Brain Res 150:543-547.

Garcia RI, Werner I, Szabo G (1979) Effect of 5-bromo-2'-deoxyuridine on growth and differentiation of cultured embryonic retinal pigment cells. In Vitro 15:779-788.

Gibb R, Kolb B (1998) A method for Golgi-Cox staining of Vibratome cut tissue. J Neurosci Methods 79:1-4.

Gould E, McEwen BS, Tanapat P, Galea LAM, Fuchs E (1997) Neurogenesis in the dentate gyrus of the adult tree shrew is regulated by psychosocial stress and NMDA receptor activation. J Neurosci 17:2492-2498.

Iball J, Morgan CH, Wilson HR (1966) Structures of 5-bromodeoxyuridine and 5-bromouridine. Nature 209:1230-1232.

Kempermann G, Kuhn HG, Gage FH (1998) Experience-induced neurogenesis in the senescent dentate gyrus. J Neurosci 18:3206-3212.

Kolb B (1987) Recovery from early cortical damage in rats. I. Differential behavioral and anatomical effects of frontal lesions at different ages of neural maturation. Behav Brain Res 25:205-220.

Kolb B, Cioe J (1996) Sex-related differences in cortical function after medial frontal lesions in rats. Behav Neurosci 110:1271-1281.

Kolb B, Whishaw IQ (1981) Decortication of rats in infancy or adulthood produced comparable functional losses on learned and species typical behaviors. J Comp Physiol Psychol 95:468-483.

Kolb B, Petrie B, Cioe J (1996) Recovery from early cortical damage in rats. VII. Comparison of the behavioural and anatomical effects of medial prefrontal lesions at different ages of neural maturation. Behav Brain Res 79:1-13.

Kolb B, Gibb R, Pedersen B, Whishaw IQ (1997) Embryonic injection of BrdU blocks later cerebral plasticity. Soc Neurosci Abstr 23:677.16.

Kolb B, Gibb R, Gorny G, Whishaw IQ (1998) Possible regeneration of rat medial frontal cortex following neonatal frontal lesions. Behav Brain Res 91:127-141.

Meller K, Breipohl W, Mestres P (1973) Effect of 5-bromodeoxyuridine on the structure of differentiating choroid plexus cells in vitro. Exp Cell Res 78:246-250.

Morris R (1980) Spatial localization does not require the presence of local cues. Learn Motiv 12:239-261.

Nagao T, Saito Y, Watanabe C, Imai K (1997) Reproductive effects of prenatal exposure to 5-bromo-2'-deoxyuridine on male rats. Reprod Toxicol 11:37-45.

Pollard DR, Baran MM, Bachvarova R (1976) The effect of 5-bromodeoxyuridine on cell division and differentiation of preimplantation mouse embryos. J Embryol Exp Morphol 35:169-178.

Prusky G, Douglas R (1998) Developmental plasticity of visual acuity in mice. Soc Neurosci Abstr 24:1876.

Ruffolo PR, Ferm VH (1965) The embryocidal and teratogenic effects of 5-bromodeoxyuridine in the pregnant hamster. Lab Invest 14:1547-1553.

Shah RM, King KO, Feeley EJ (1991) Pathogenesis of bromodeoxyuridine-induced cleft palate in hamster. Am J Anat 190:219-230.

Stewart J, Kolb B (1988) The effects of neonatal gonadectomy and prenatal stress on cortical thickness and asymmetry in rats. Behav Neural Biol 49:344-360.

Stockdale F, Okazaki K, Nameroff M, Holtzer H (1964) 5-Bromodeoxyuridine: effect on myogenesis in vitro. Science 146:533-535.

Sutherland RJ, Whishaw IQ, Kolb B (1983) A behavioral analysis of spatial localization following electrolytic, kainate-, or colchicineinduced damage to the hippocampal formation in the rat. Behav Brain Res 7:133-153.

Trencer R, Brachet J (1973) Studies on the effects of bromodeoxyuridine (BudR) on differentiation. Differentiation 1:51-64.

Webster W, Shimada M, Langman J (1973) Effect of fluorodeoxyuridine, colcemid, and bromodeoxyuridine on developing neocortex of the mouse. Am J Anat 137:67-86.

Whishaw IQ, O'Connor RB, Dunnett SB (1986) The contributions of the motor cortex, nigrostriatal dopamine and caudate-putamen to skilled forelimb use in the rat. Brain 109:805-843.

Wilt FH, Anderson M (1972) The action of 5-bromodeoxyuridine on differentiation. Dev Biol 28:443-447.

Wrathall JR, Oliver C, Silagi S, Essner E (1973) Suppression of pigmentation in mouse melanoma cells by 5-bromodeoxyuridine: effects on tyrosinase activity and melanosome formation. J Cell Biol 57:406-423.

Younkin L, Silberberg D (1973) Myelination in developing cultured newborn rat cerebellum inhibited by 5-bromodeoxyuridine. Exp Cell Res 76:455-458.

Yu WHA (1976) The effect of 5-bromodeoxyuridine on the postnatal development of the rat cerebellum: a biochemical study. Brain Res 118:281-291.

Zimmerman J, Brumbaugh J, Biehl J, Holtzer H (1974) The effect of 5-bromodeoxyuridine on the differentiation of chick embryo pigment cells. Exp Cell Res 83:159-165. 